\title{
Themenschwerpunkt „Women‘s Mental Health“
}

\section{Liebe Leserinnen und Leser,}

Viele psychische Erkrankungen zeigen geschlechtsspezifische Besonderheiten, etwa in der Inzidenz und Prävalenz, aber auch in den Risikofaktoren und pathogenetischen Mechanismen. Moderne Psychiatrie berücksichtigt dies, nicht nur in der Diagnostik, sondern auch in einer geschlechtersensiblen Therapie.

Die zunehmende Bedeutung einer geschlechtersensiblen Psychiatrie und Psychotherapie wurde in den angloamerikanischen Ländern schon lange erkannt, und auch in den deutschsprachigen Ländern gibt es ein wachsendes Interesse daran. Ausdruck dieser Entwicklung ist unter anderem die Gründung zweier Gesellschaften, die sich mit dieser Thematik auf interdisziplinärer Basis beschäftigen.

Zum einen ist dies die Gesellschaft für die psychische Gesundheit von Frauen (GPGF), die wir 1999 in Basel gegründet haben und die seither regelmässig Kongresse in den drei deutschsprachigen Ländern Deutschland, Schweiz und Österreich abhält, zuletzt im September 2011 in Frankfurt unter dem Thema „Geschlecht-Gender-Psyche“. Präsidentin ist neu Frau Prof. Beate WimmerPuchinger aus Wien, wo auch der 11. Kongress der GPGF im Herbst 2012 stattfinden wird. Für erste Vorankündigungen besuchen Sie bitte unsere Website http://www.gpgf.org/.

Die andere, internationale Gesellschaft haben wir im Jahr 2000 in Berlin gegründet, die International Association for Women's Mental Health (IAWMH).
Sie hatte im März diesen Jahres ihren 4. Kongress in Madrid und wird den nächsten Kongress vom 4. bis 7. März 2013 in Lima, Peru, abhalten (http:// www.iawmh.org/).

Das Ziel der IAWMH ist es, die psychische Gesundheit von Frauen weltweit $\mathrm{zu}$ verbessern. Unser Ansatz ist dabei ein gezielt interdisziplinärer. In der $\mathrm{Ge}$ sellschaft sind nicht nur PsychiaterInnen und PsychotherapeutInnen, PsychologInnen, SoziologInnen und MedizinerInnen vertreten, sondern auch GrundlagenforscherInnen, EndokrinologInnen, BiologInnen, HistorikerInnen, PolitikerInnen, PhilosophInnen etc. Ziel ist es, die psychische Gesundheit in ihrer ganzen bio-psycho-sozialen Komplexität zu verstehen, auf dieser Basis zu diagnostizieren und zu behandeln, vor allem aber auch prophylaktisch zu wirken, also psychische Krankheit zu verhindern.

Die Kongresse beschäftigen sich deshalb nicht nur mit den psychosozialen, ökonomischen und kulturellen Bedingungen von Frauengesundheit, sondern auch mit biologischen Aspekten wie Geschlechtsunterschieden des Gehirns, hormonellen oder genetischen Einflüssen und vielem mehr. KlinikerInnen und ForscherInnen verschiedener Disziplinen versuchen, ihr Wissen zusammenzutragen und zu diskutieren, um innovative Entwicklungen zur Verbesserung der psychischen Gesundheit von Frauen weltweit anzustossen.

Innerhalb der IAWMH gibt es verschiedene Arbeitsgruppen, die sich mit spe- zifischen Themen wie „Education“, „Policy and Ethics“, „, Research and Scientific Program" beschäftigen. Bei Interesse besuchen Sie unsere Websites und schreiben Sie uns. In beiden Gesellschaften ist die Mitgliedschaft derzeit noch kostenlos. Von der IAWMH erhalten Sie regelmässig unsere Newsletter und werden über alle Aktivitäten informiert. Auch können Sie sich bald zu unseren nächsten Kongressen anmelden - mit eigenen aktuellen Beiträgen, oder aber einfach zum Zuhören und Mitdiskutieren. Ich würde mich über Ihr Interesse sehr freuen.

Prof. Dr. med. Anita Riecher-Rössler Präsidentin der IAWMH und Gründungspräsidentin der GPGF

Chefärztin der Psychiatrische Universitätspoliklinik der Universitären Psychiatrischen Kliniken Basel, Schweiz

\section{Links:}

Gesellschaft für die psychische Gesundheit von Frauen (GPGF): www.gpgf.org

International Association for Women's Mental Health: http://www.iawmh.org/ 